\title{
SUPPURATIVE LESIONS OF THE BRAIN-STEM
}

\author{
BY \\ ALLAN HULME \\ From the South-Western Regional Neurosurgical Unit, Frenchay Hospital, Bristol
}

In contrast to the frequency with which abscesses occur elsewhere in the brain, suppuration confined to, or extending into, the brain-stem is rare.

Cassirer (1902) reported one case of pontine abscess and abstracted 10 from the literature. Further cases were added by Bregman (1906), Lichtenstein and Zeitlin (1936), Gaida (1939), and Roger, Poursines, Roger, and Tamalet (1951). Richland (1952) traced 15 cases in the literature and added a further one; of these, five were attributed to direct spread of infection from the ear, while the remainder were of metastatic origin. A further instance was reported by Sutin (1958).

Weickhardt and Watts (1944), describing a case of abscess in the medulla oblongata, found nine previously recorded instances, of which only three, those of Schlesinger (1894), Moniz (1934), and Norman (1941), were confined to the medulla. In their own and other cases the lesion extended into the pons or upper cervical spinal cord. Six cases were derived from distant foci and two from the ear. The origin of the remainder was obscure, though one, that of Abercrombie (1836), is thought to have been a tuberculoma.

As would be expected, the neurological manifestations vary widely, and change with progression of the pathological process, according to the cranial nerve nuclei and fibre tracts involved. Commonly, fairly accurate anatomical localization is possible.

In all reported cases, the condition has progressed, usually in a matter of days or weeks to a fatal termination. Scalabrino and Clivio (1951) reported recovery following systemic and intrathecal penicillin therapy in a case of presumed metastatic suppurative inflammation in the midbrain. The diagnosis, however, appears not to have been established with certainty. The following cases illustrate the difficulties encountered in diagnosing this condition, even with the aid of contrast radiography.

\section{Case Reports}

Case 1.-N.D.P., a 40-year-old woman, presented with an 11-day history of anorexia, and one week's history of increasingly severe frontal headache accompanied by vomiting, pyrexia, and drowsiness. Three days after the onset of headache, slurring of speech and weakness of the right upper limb were noted.

On admission she was dehydrated, febrile (temperature $101 \cdot 2^{\circ} \mathrm{F}$.), and restless. She was unable to speak, and the right limbs were paralysed and flaccid. No facial paresis was noted. The ocular fundi showed bilateral early papilloedema, and she was thought to have a right homonymous hemianopia.

There was moderate neck rigidity. The findings indicated a lesion of the left cerebral hemisphere but carotid angiography showed no displacement of vessels or other abnormality.

Cerebrospinal fluid examination performed on seven occasions revealed a moderate pleocytosis, varying between 86 and 350 leucocytes per c.mm., with a preponderance of lymphocytes. Protein ranged between 60 and $228 \mathrm{mg}$. per $100 \mathrm{ml}$.; 20,000 units of penicillin were instilled intrathecally on four occasions. After transient improvement her condition deteriorated further, with deepening coma and development of a right oculomotor palsy and right facial paresis. Ventriculography showed mild ventricular dilatation; the third and fourth ventricles and aqueduct were normally situated, but air failed to emerge from the fourth ventricle into the basal cisterns, suggesting obstruction due to inflammatory adhesions in the region of the foramen of Magendie. Continuous ventricular drainage was instituted. Further progression of neurological dysfunction, including paresis of the left lower limb and a left external rectus palsy preceded death, 17 days after the onset of her illness.

At necropsy, the principal pathological findings outside the cranial cavity were acute cholecystitis and bilateral patchy bronchopneumonia.

The cerebral hemispheres and cerebellum appeared normal on inspection but the pons had a greyish pink appearance and was extensively softened. Serial sections of the hardened brain showed discoloration and softening, mainly in the right side of the pons, extending into the midbrain as far as the substantia nigra, across the midline and downwards to the upper end of the medulla. A few areas of yellow pus were seen in the softened area. Histological examination showed suppurative encephalitis with multiple early abscess formation.

Case 2.-Mrs. V.K., aged 43 years, gave a history of recent exacerbation of bronchitis, with mucopurulent sputum. Over a period of three days she had developed numbness and weakness of the left limbs, starting with 
tingling in the hand, and accompanied by occipital and post-nuchal headache. During the same period she had noted diplopia, slurring of speech, and difficulty in breathing.

On admission she was obviously ill, and there were signs of bronchopneumonia. Neurological examination revealed paresis of the right external rectus muscle, impaired palatal movement, and deviation of the tongue to the right on protrusion. The left limbs were paretic and the left plantar response extensor.

The cerebrospinal fluid was under a pressure of 140 mm., and contained 2 white cells per c.mm., protein $85 \mathrm{mg}$. per $100 \mathrm{ml}$, with increased globulin, and sugar $52 \mathrm{mg}$. per $100 \mathrm{ml}$.

Two days later, she showed impairment of all conjugate eye movements, particularly to the right, and slight skew deviation of the eyes, the left being a little up and the right a little down. The right pupil was a little larger than the left, but both reacted to light. Hypalgesia of the left limbs, trunk, and face was noted and right corneal sensation also seemed to be diminished. In addition to the paresis of the left limbs, there was a well-marked right facial weakness of lower motor neurone type. She was markedly deaf in the right ear, but there were no signs of aural infection.

The clinical signs suggested a progressive lesion in the brain-stem and contrast air studies were advised. No air entered the ventricles on attempted lumbar air encephalography, but subsequent ventriculograms appeared normal; in particular there was no apparent distortion or obstruction of the third or fourth ventricles.

A second cerebrospinal fluid examination revealed 65 white cells per c.mm. (16\% polymorphonuclear leucocytes and $84 \%$ lymphocytes); protein $50 \mathrm{mg}$. per $100 \mathrm{ml}$. and sugar $97 \mathrm{mg}$. per $100 \mathrm{ml}$.

Electroencephalography gave a record barely outside the normal range of variation and with no localizing features.

Culture of the sputum yielded a pneumococcus which was sensitive to tetracycline and treatment with the appropriate antibiotic was given. Anticoagulants were administered on the assumption that the cerebral lesion was a vascular thrombosis. Her condition deteriorated, however, and she died 16 days after admission to hospital.

At necropsy a solitary abscess containing thick green pus was found in the right half of the pons. It measured $1.5 \mathrm{~cm}$. from above down, $2.2 \mathrm{~cm}$., antero-posteriorly, and $1.8 \mathrm{~cm}$. transversely. It bulged into the floor of the fourth ventricle but had not ruptured (Fig. 1).

Case 3.-Mrs. A.M., aged 41 years, had suffered from migrainous attacks for many years. Four days before admission she awoke with frontal headache and seemed dazed and clumsy in her movements. She appeared to see more clearly with one eye closed, possibly due to diplopia. Some hours later she suddenly became unconscious and on admission to hospital she was comatose with dilated non-reacting pupils, spasticity of all limbs, and periodic respiration. During the next four days her condition remained essentially unchanged, though the level of consciousness improved slightly, respiratory

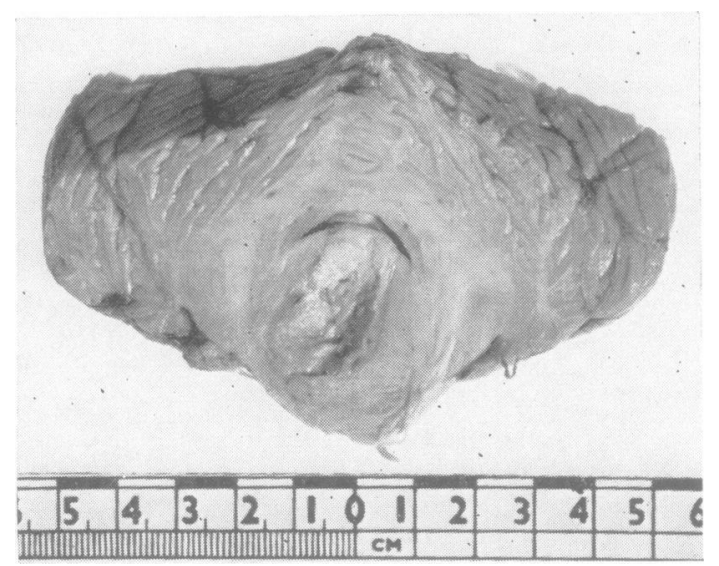

FIG. 1.-Transverse section of pons in Case 2 showing abscess cavity.

rhythm returned to normal, and the limbs became flaccid. The plantar responses remained extensor.

Blood examination revealed moderate anaemia $(\mathrm{Hb}$ $57 \%$ ) and 28,200 white cells per c.mm. (90\% polymorphonuclear leucocytes). Bilateral carotid and vertebral angiograms provided no evidence of a space-occupying lesion or of arterial occulsion. The electroencephalogramb showed delta activity from all areas of the brain with frequent monophasic complexes. The cerebrospinal fluig was normal in all respects.

A tentative diagnosis of brain-stem thrombosis was made. A blood transfusion was given to combat anaemia the haemoglobin level having fallen as low as $42 \%$. The patient was transferred to another hospital where she died two weeks later. Necropsy revealed bilaterá bronchiectasis and a chronic multilocular abscess in the left lung.

Examination of the fresh brain suggested that there were two metastatic abscesses, one presenting on the upper surface of the right cerebral hemisphere at the junction of its anterior two-thirds and posterior one-third, and a second on the right side of the midbrain. Serial sections of the hardened brain showed that in fact there was one large communicating cavity. The right cerebral peduncle was largely destroyed. A coagulase-positive Staphylococcus aureus was isolated from the abscess cavity. A similar organism had been obtained from the sputum during life.

\section{Comment}

In all three cases the abscess was of metastatic origin and probably derived from the lungs. The syndrome was of sudden or rapid onset, suggesting an embolic phenomenon and the clinical pattern changed rapidly as the lesion developed.

In Cases 2 and 3, the neurological signs localized the pathological process to the brain-stem, but in Case 1 the initial syndrome of right hemiplegia, aphasia, and probable right hemianopia implicated 
the left cerebral hemisphere; only later did contralateral signs suggest pathology at a lower level. A possible explanation might be that an early metastatic lesion in the left cerebral hemisphere was quelled with antibiotics and that a further embolus occurred later in the brain-stem. It is noteworthy that the inflammatory process here was at an early stage; the multiple foci of suppuration had not localized into a single cavity nor had encapsulation occurred. In Case 3 the early and rapid onset of coma and pupillary dilatation suggests involvement of the midbrain, and it seems likely that there were embolic foci here and in the right cerebral hemisphere which later coalesced to form a single cavity.

In no case was assistance in localization obtained from contrast radiography.

In Case $\mathbf{2}$ it is surprising that no distortion of the fourth ventricle shadow was apparent, despite the protrusion into the floor caused by the underlying mass (Fig. 1). It is possible that ventriculography using an opaque medium, such as Myodil, would have given more precise definition and perhaps demonstrated a deformity.

Only in Case 1 did the cerebrospinal fluid show an early pleocytosis, suggestive of a meningeal reaction.

Electroencephalography also proved unhelpful in localization.

Various authors stress the fact that embolic phenomena occur much more commonly in the distribution of the middle cerebral than of other intracranial arteries (Richland, 1952; Stuart, O'Brien, and McNally, 1955). It is presumably the relative invulnerability of the vertebral basilar system which accounts for the rarity of metastatic suppuration in the brain-stem.

Gowers (1893) found only three instances of pontine and one of medullary localization among 231 brain abscesses, and Le Fort and Lehman, quoted by Cassirer (1902), six in a series of 458 .

The three cases described above occurred in a series of 105 instances of intracranial abscess during a period of 11 years. The incidence thus appears to be less than $3 \%$.

Hitherto the diagnosis has been made only at necropsy; it seems unlikely, however, that any effective surgical intervention would be practicable even if the condition were recognized during life and its rarity is indeed fortunate.

\section{Summary}

Three cases of suppuration in the brain-stem are described and the literature briefly reviewed.

The condition is rare and the diagnosis difficult, since special radiological techniques appear to give little assistance.

\section{REFERENCES}

Abercrombie, J. (1836). Pathological and Practical Researches on Diseases of the Brain and Spinal Cord, 3rd ed., p. 103. Maclachlan, Stewart, Edinburgh.

Bregman, L. (1906). Dtsch. Z. Nervenheilk., 31, 86.

Bregman, L. (1906). Dtsch. Z. Nervenheilk., 31, 86.

Gasirer, R. (1902). Arch. Psychiat. Nervenkr., 36, 153.

Gowers, W. R. (1893). A Manual of Diseases of the Nervous System, 2nd ed., Vol. 2 , p. 473. Churchill, London.

Lichtenstein, B. W., and Zeitlin, H. (1936). J. Amer. med. Ass., 106, 1057.

Moniz, E. (1934). Rev. Oto-neuro-ophtal., 12, 568.

Norman, H. B. (1941). Brit med. J, 1, 403.

Richland, K. J. (1952). Bull. Los Angeles neurol. Soc., 17, 122

Roger, H., Poursines, Y Bull. Los Angeles neurol. Soc., 17, 122. neurol., 84, 246.

Scalabrino, R., and Clivio, C. (1951). Rev. Oto-neuro-oftal.(B. Aires), 26, 495 .

Schlesinger, H. (1894). Arb. Inst. Anat. Physiol. Centralnervensyst. Wien Univ., 2, 114.

Stuart, E. A., O'B̈rien, F. H., and McNally, W. J. (1955). A.M.A. Arch. Otolaryng., 61, 212.

Sutin, M. I. (1958). Vop Nejrohir., 6, 43-44 (Abst. Sovetsk. Med., Pt B, 4, No. 1, 1960, Abst. No. 295)

Weickhardt, G. D., and Watts, J. W. (1944). Arch. Neurol. Psychiat. (Chicago), 51, 282. 